\title{
Properties of polycyclic aromatic hydrocarbons in the star forming environment in nearby galaxies
}

\author{
Itsuki Sakon ${ }^{1}$, Takashi Onaka ${ }^{1}$, Daisuke Kato ${ }^{1}$, \\ Hidehiro Kaneda ${ }^{2}$, Hirokazu Kataza ${ }^{2}$, Yoko Okada ${ }^{2}$, \\ Akiko Kawamura ${ }^{3}$, Toshikazu Onishi ${ }^{3}$, and Yasuo Fukui ${ }^{3}$ \\ ${ }^{1}$ Department of Astronomy, Graduate School of Science, University of Tokyo, \\ 7-3-1 Hongo, Bunkyo-ku, Tokyo 113-0033, Japan \\ email: isakon@astron.s.u-tokyo.ac.jp \\ ${ }^{2}$ Institute of Space and Astronautical Science, Japan Aerospace Exploration Agency, \\ 3-1-1 Yoshinodai, Sagamihara, Kanagawa 229-8510, Japan \\ ${ }^{3}$ Department of Astrophysics, Nagoya University, Chikusa-ku, Nagoya 464-8602, Japan
}

\begin{abstract}
We have carried out the mid-infrared slit spectroscopic observations of sources in the LMC and in NGC 6946 with AKARI/IRC. We investigate the properties of the UIR bands in terms of the star forming activities. We find systematically larger ratios of UIR bands in 6-9 $\mu \mathrm{m}$ to $11.2 \mu \mathrm{m}$ band in active star forming regions than in the quiet regions. This behavior is consistent with the photo-ionization model of PAHs. Our results suggest that the ratios of UIR bands in $6-9 \mu \mathrm{m}$ to $11.2 \mu \mathrm{m}$ band can be used as more efficient and vigorous tools to measure the extent of on-going star formation in remote galaxies rather than just the presence or absence of the features themselves.
\end{abstract}

Keywords. Dust, extinction, ISM: lines and bands, galaxies: ISM, infrared: ISM

\section{Introduction}

Ionization of interstellar polycyclic aromatic hydrocarbons (PAHs) is one of the most notable processes to cause the variations in the UIR band spectra. Many studies based on quantum chemical calculations as well as laboratory experiments have shown that the strengths of the PAH features in 6-9 $\mu \mathrm{m}$ relative to $11.2 \mu \mathrm{m}$ feature increase when PAHs are ionized (Allamandola et al. 1985, Hudgins \& Allamandola 1999, DeFrees et al. 1993). Actually several recent observations have reported the decreasing band ratios of $7.7 \mu \mathrm{m} / 11.2 \mu \mathrm{m}$ and $8.6 \mu \mathrm{m} / 11.2 \mu \mathrm{m}$ with the increasing distance from the heating source within the reflection nebulae NGC 1333 (Bregman \& Temi 2005, Joblin et al. 1996) and also the higher 6-9 $\mu \mathrm{m}$ bands to $11.2 \mu \mathrm{m}$ band ratios for the Herbig $\mathrm{Ae} / \mathrm{Be}$ stars with earlier stellar spectral types (Sloan et al. 2005), which are reasonably explained by the ionization model of PAHs.

In this proceedings, we present some of our recent observational results on mid-infrared spectroscopy of interstellar PAHs in Large Magellanic Cloud (LMC) and in the nearby starforming galaxy NGC 6946 using Infrared Camera (IRC, Onaka et al. 2007) onboard AKARI. 


\section{UIR bands in the LMC}

As for the AKARI/IRC spectroscopy of interstellar PAHs in the LMC, we selected two slit positions at $\left(\alpha_{J 2000}, \delta_{J 2000}\right)=\left(5^{h} 39^{m} 57^{s} .4,-69^{d} 45^{m} 29^{s}\right),\left(5^{h} 38^{m} 19^{s} .9,-70^{d} 07^{m} 26^{s}\right)$; The former one corresponds to the molecular clouds associated with massive young star clusters near N159 (Bica et al. 1996) and the latter one corresponds to the CO molecular cloud without young star clusters. The length of the slit for mid-infrared spectroscopy is about $\sim 40$ ". Two regions, 1 and 2, are defined within the former slit position and one region 3 is defined within the latter slit position. The zodi-subtracted spectra at regions 1, 2 and 3 are shown in Figure 1. All the spectra show a series of UIR bands at $6.2 \mu \mathrm{m}$, $7.7 \mu \mathrm{m}, 8.6 \mu \mathrm{m}$ and $11.2 \mu \mathrm{m}$. The spectra at regions 1 and 2 show the ionic lines of [Ar III] at $8.99 \mu \mathrm{m},[\mathrm{S} \mathrm{IV}]$ at $10.51 \mu \mathrm{m}$ and [Ne II] at $12.81 \mu \mathrm{m}$ showing that the ISM at regions 1 and 2 is powered by massive stars. On the other hand, the spectrum at region 3 shows no signs of those ionic lines showing that the ISM at region 3 is powered by less energetic photons and is in quiet environment.

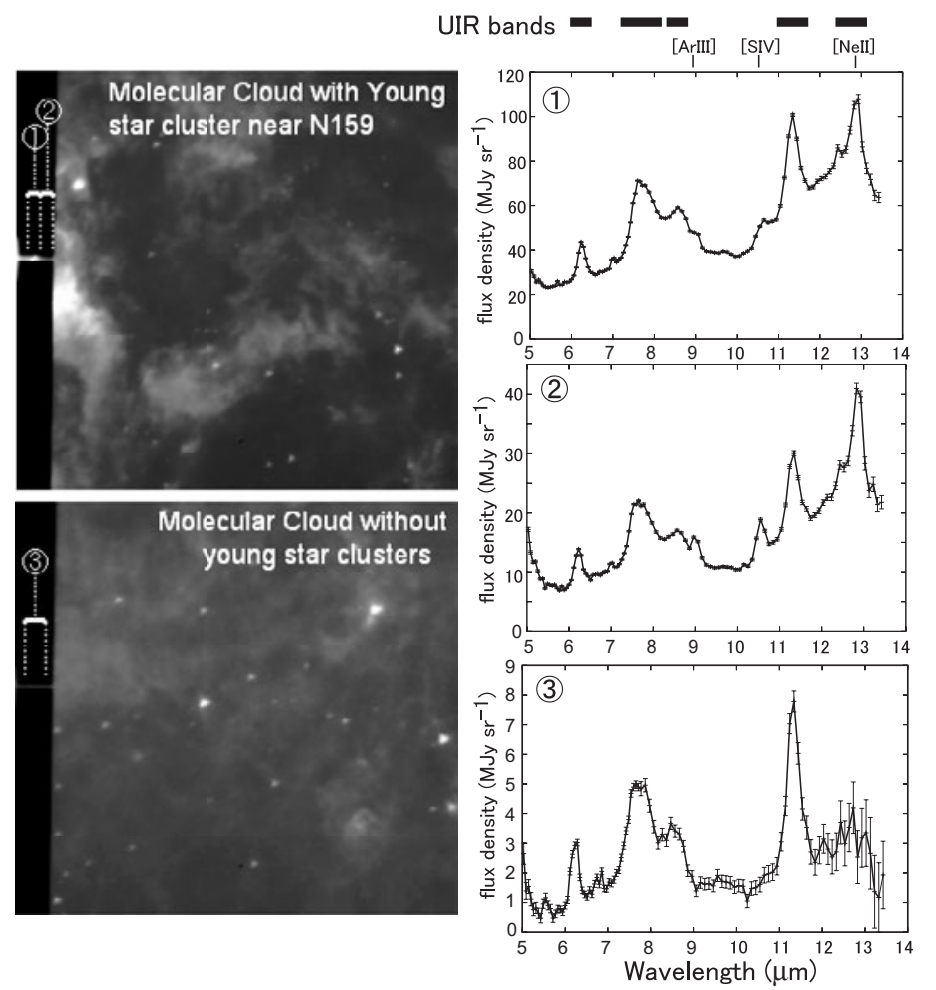

Figure 1. Locations of the slit for mid-infrared spectroscopy of sources in the LMC and the zodi-subtracted spectra of regions 1,2 and 3, respectively.

We measure the strengths of UIR band features by assuming simple baselines through continuum points at $6.0 \mu \mathrm{m}, 6.5 \mu \mathrm{m}, 7.2 \mu \mathrm{m}, 8.2 \mu \mathrm{m}, 8.8 \mu \mathrm{m}, 10.9 \mu \mathrm{m}$ and $11.7 \mu \mathrm{m}$. The UIR band ratios of $6.2 \mu \mathrm{m} / 11.2 \mu \mathrm{m}, 7.7 \mu \mathrm{m} / 11.2 \mu \mathrm{m}$, and $8.6 \mu \mathrm{m} / 11.2 \mu \mathrm{m}$ are summarized in Table 1 . We find systematically larger ratios of the UIR bands in $6 \mu \mathrm{m}-$ $9 \mu \mathrm{m}$ to UIR $11.2 \mu \mathrm{m}$ at regions 1 and 2 than at region 3, which is consistent with the photo-ionization model of interstellar PAHs. 
Table 1. Relative band strengths of the UIR features

\begin{tabular}{|c|c|c|c|}
\hline Region Identification $^{1}$ & UIR $6.2 \mu \mathrm{m} / 11.2 \mu \mathrm{m}$ & UIR $7.7 \mu \mathrm{m} / 11.2 \mu \mathrm{m}$ & $8.6 \mu \mathrm{m} / 11.2 \mu \mathrm{m}$ \\
\hline region 1 & $1.27 \pm 0.11$ & $3.44 \pm 0.27$ & $0.46 \pm 0.07$ \\
\hline region 2 & $2.05 \pm 0.24$ & $5.31 \pm 0.60$ & $0.60 \pm 0.12$ \\
\hline region 3 & $0.76 \pm 0.11$ & $1.80 \pm 0.21$ & $0.29 \pm 0.11$ \\
\hline
\end{tabular}

Notes:

1 Regions 1 and 2 correspond to active regions associated with young star clusters near N159 while region 3 corresponds to quiet region in the molecular ridge.

\section{UIR bands in the nearby starforming galaxy NGC 6946}

Similar results are obtained for the AKARI/IRC spectroscopic observation of interstellar PAHs in the nearby star-forming galaxy NGC 6946. The slit for mid-infrared spectroscopy was laid so that it covers both the star forming region LDG source 47 at $\left(\alpha_{J 2000}, \delta_{J 2000}\right)=\left(20^{h} 34^{m} 50^{s} .80,+60^{d} 07^{m} 48^{s} .9\right)$ (Hyman et al. 2000$)$ located on a galactic arm and the quiet region in the interarm. The obtained spectra are shown in Figure 2. In the same way, we measured the strength of each UIR band by assuming a local linear continuum as described in $\S 2$ and investigate the UIR band ratios of $6.2 \mu \mathrm{m} / 11.2 \mu \mathrm{m}$, $7.7 \mu \mathrm{m} / 11.2 \mu \mathrm{m}$, and $8.6 \mu \mathrm{m} / 11.2 \mu \mathrm{m}$ (see Table 2). Even if we take account of the 3 magnitudes of visual extinction towards the arm region and the LDG source 47 (Ferguson et al. 1998), we find those ratios have systematically larger values in the star forming region on the galactic arm than in the quiet region in the interarm.
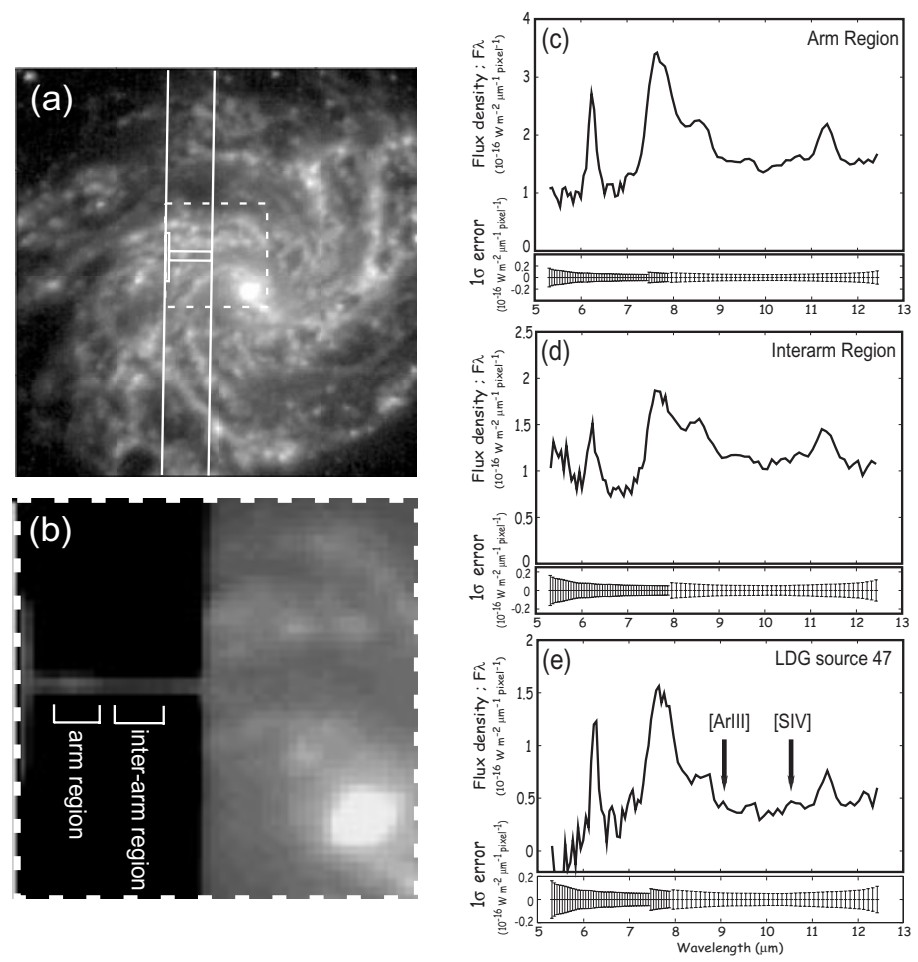

Figure 2. (a)(b) Locations of the slit for mid-infrared spectroscopy of sources in NGC 6946 . $(c)(d)(e)$ Obtained mid-infrared spectra of the arm region, the interarm region and the star forming region LDG source 47, respectively. The spectrum of (e) is simply made by (c)-(d). 
Table 2. Relative band strengths of the UIR features

\begin{tabular}{|c|c|c|c|}
\hline Region Identification & UIR $6.2 \mu \mathrm{m} / 11.2 \mu \mathrm{m}$ & UIR $7.7 \mu \mathrm{m} / 11.2 \mu \mathrm{m}$ & $8.6 \mu \mathrm{m} / 11.2 \mu \mathrm{m}$ \\
\hline NGC 6946 Interarm $^{1}$ & $0.84 \pm 0.14$ & $2.27 \pm 0.32$ & $0.50 \pm 0.14$ \\
\hline NGC $6946 \mathrm{Arm}^{2}$ & $1.33 \pm 0.13$ & $3.32 \pm 0.35$ & $0.56 \pm 0.09$ \\
\hline NGC 6946 LDG source $47^{2}$ & $2.36 \pm 0.64$ & $5.51 \pm 1.44$ & $0.66 \pm 0.28$ \\
\hline
\end{tabular}

Notes:

1 No extinction correction are undertaken for the extreme case.

2 Extinction of $A_{V}=3 \mathrm{mag}$ (Ferguson et al. 1998) is taken into account.

\section{Discussion and Summary}

Based on the mid-infrared spectroscopic observations of sources in the LMC and in NGC 6946 with AKARI/IRC, we investigate the properties of the UIR bands in terms of the star forming activities. We find systematically larger ratios of UIR bands in 6 $9 \mu \mathrm{m}$ to $11.2 \mu \mathrm{m}$ band in active star forming regions than in the quiet regions. This behavior is consistent with the photo-ionization model of interstellar PAHs. On the other hand, the changing in size distribution of PAHs should also be an important evolution process of interstellar PAHs in active star forming environment. Our obtained spectra have shown typically larger UIR band to underlying plateau ratio in the star forming regions than in the quiet regions (Sakon et al. 2007). Since the UIR bands are supposed to be carried by PAHs made of 20-100 carbon atoms while the underlying plateau emission by PAH clusters made of more than 500 carbon atoms (Allamandola et al. 1989), photoevaporation of PAH clusters into free-flying PAHs in the star forming region may explain the larger ratio of UIR bands to plateau emission in the star forming region than in the quiet region consistently. If the photo-evaporation of PAH clusters into free-flying PAHs as well as the photo-ionization of PAHs are the major evolutional processes of interstellar PAHs in an active star forming environment, our present results suggest that the ratios of UIR bands in 6-9 $\mu \mathrm{m}$ to $11.2 \mu \mathrm{m}$ band can be used as more efficient and vigorous tools to measure the extent of on-going star formation in remote galaxies rather than just the presence or absence of the features themselves.

$A K A R I$ is a JAXA project with the participation of ESA. We thank all the members of the AKARI project, particularly those who have engaged in the observation planning and the satellite operation during the performance verification phase, for their continuous help and support. This work is supported in part by a Grant-in-Aid for Scientific Research on Priority Areas from the Ministry of Education, Culture, Sports, Science, and Technology of Japan and Grants-in-Aid for Scientific Research from the JSPS.

\section{References}

Allamandola, L. J., Tielens, A. G. G. M., \& Barker, J. R. 1985, ApJ (Letters), 290, L25

Allamandola, L. J., Tielens, A. G. G. M., \& Barker, J. R. 1989, ApJS, 71, 733

Bica, E., Claria, J. J., Dottori, H., Santos, J. F. C. Jr., \& Piatti, A. E. 1996, ApJS, 102, 57

Bregman, J. \& Temi, P. 2005, ApJ, 621, 831

de Frees, D. J., Miller, M. D., Talbi, D., Pauzat, F., \& Ellinger, Y. 1993, ApJ, 408, 530

Ferguson, A. M. N., Gallagher, J. S., \& Wyse, R. F. G. 1998, AJ, 116, 673

Hudgins, D. M. \& Allamandola, L. J. 1999, ApJ (Letters), 516, 41

Hyman, S. D., Lacey, C. K., Weiler, K. W., \& Van Dyk, S. D. 2000, AJ, 119, 207

Joblin, C., Tielens, A. G. G. M., Geballe, T. R., \& Wooden, D. H. 1996, ApJ (Letters), 460, L119

Onaka, T., et al. 2007, PASJ, 59, S401

Sloan, G. C., et al. 2005, ApJ, 632, 956,

Sakon, I., et al. 2007, PASJ, 59, S483 


\section{Discussion}

SLOAN: I just want to compliment you on the 7.6-7.8 $\mu$ m plateau analysis. I've been asking J.D. Smith for a long time to do exactly this kind of analysis and I am delighted to see someone doing it. Thanks.

SAKON: I think the size effect on the PAH band ratio as well as the ionization effect on the UIR band ratios are quasi coupled, so we need to make further observations as well as laboratory the experiments. Such kind of collaboration will be very important for the understanding of the interstellar UIR bands.

UNKNOWN: In your spectra, the broadband continuum may be greater than the small spectral peaks you're measuring. In fact, you could be buried some of the spectral peaks in the continuum. So what is the emission mechanism that gives you this broadband continuum?

SAKON: The continuum could be due to very small grains excited by ultraviolet photons and stochastically heated to a certain temperature. The carrier can be a combination of various kinds of molecules including PAHs, QCC, or aliphatic materials. The detailed modeling for the infrared emission properties of such kind of materials is very difficult.

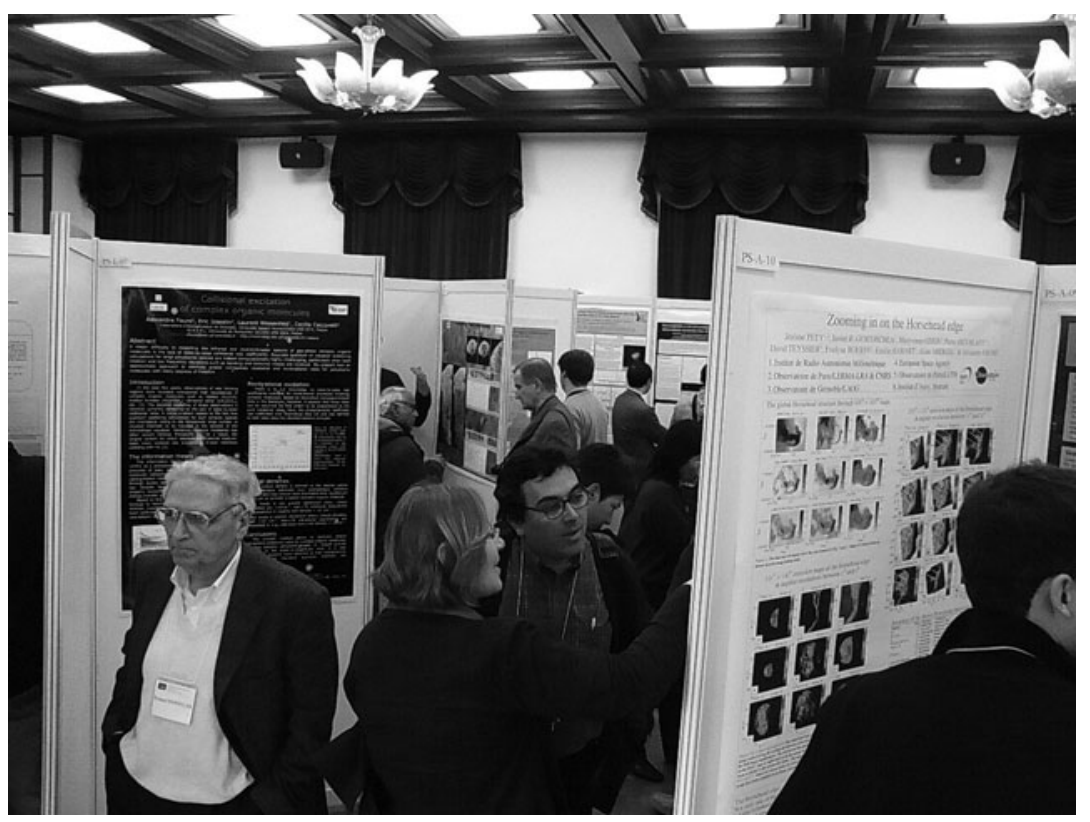

Poster session at the convocation room (in the foreground from left to right: Renauld Papoular, Yolanda Gomez and Arturo Manchado). 


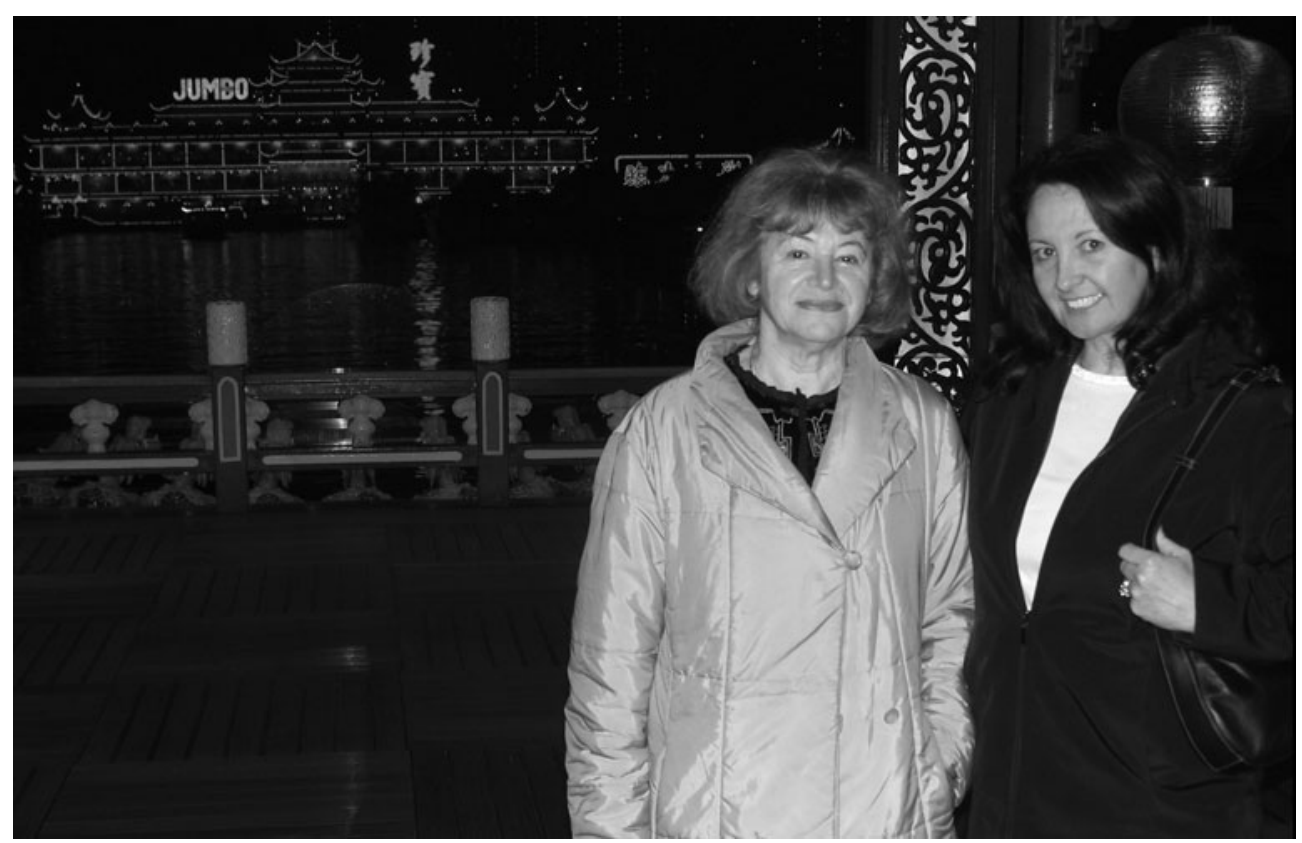

Catherine Cesarsky (left) and Yvonne Pendleton (right) by the Jumbo floating restaurant in Aberdeen (photo by Dale Cruikshank). 